\title{
Investigating the Influence of Bending Parameters on the Springback Behavior of Ultra-High Strength Spring Strips
}

\author{
Karsten Richter ${ }^{1, a^{*}}$, Franz Reuther ${ }^{1, b}$, Roland Müller ${ }^{1, c}$ and Dirk Landgrebe $e^{1, d}$ \\ ${ }^{1}$ Fraunhofer Institute for Machine Tools and Forming Technology IWU, Reichenhainer Str. 88, \\ 09126 Chemnitz, Germany \\ ${ }^{a}$ karsten.richter@iwu.fraunhofer.de, ${ }^{b}$ franz.reuther@iwu.fraunhofer.de, \\ croland.mueller@iwu.fraunhofer.de, dirk.landgrebe@iwu.fraunhofer.de
}

\begin{abstract}
Keywords: spring strips, springback, springback dispersion, stamping-bending technology,
\end{abstract} bending, process parameters, resource efficiency

\begin{abstract}
The spring industry has to process ultra-high strength spring strips of the steel grade 1.4310 (X10CrNi18-8). Springback during bending is a limiting factor for resource-efficient production processes in the so-called stamping bending technology. This paper reports a mainly experimental investigation on the influence of bending parameters on the evolving springback of spring strips of the grade 1.4310 with tensile strengths $T S=1500-1800 \mathrm{MPa}$.
\end{abstract}

\section{Introduction}

In spite of the rather small dimensions common for springs, the trend towards lightweight design has reached spring manufacturers as well. Further weight reduction and miniaturization of springs can only be reached by substituting the spring strips with those of even smaller thicknesses and even higher strengths. Prior to the 1950s, strips of the chromium-nickel-steel 1.4301 (X5CrNi18-10) were almost exclusively processed to springs made of stainless steels. Yet, tensile strengths are limited to approximately $T S=1500 \mathrm{MPa}$, with ultimate elongations merely exceeding $U E=1-2 \%$. In order to overcome these limitations, the steel grade 1.4310 (X10CrNi18-8) with highly beneficial material properties was developed. At a tensile strength $T S=1500 \mathrm{MPa}$, ultimate elongations of approximately $U E=10-12 \%$ remain available. Up to now, tensile strengths of up to $T S=2100$ $2200 \mathrm{MPa}$ have been reached with sheet thicknesses available as low as $t=0.05 \mathrm{~mm}$. Neither press hardening nor micro-alloyed steels, which are regularly processed with hot or cold forming technologies in the automotive industry, can compete with these strengths [1]. Thus, spring manufacturing can be considered the technology that has to deal with the highest sheet strengths of all the metal forming industries. The necessity to use materials within the stated range of strength is based on the functionality springs have to fulfil. Depending on the specifics of the application, a selection of suitable highly elastic materials is mandatory, allowing for large elastic deformations and suitable forms $[2,3]$.

The production of these spring strips is realized by cold rolling where several steps of rolling and heat treatment are combined to reach the desired properties. The enhancement of strength is closely connected to the reduction of sheet metal thickness. During this treatment, the microstructure of the metastable austenitic stainless steel 1.4310 is set by generating a strain-induced martensite providing the strength and hardness of the material and by setting residual stress and surface conditions of the strip. The formerly face-centered cubic ferritic space-lattice of the $\gamma$-phase in annealed condition is transformed into forced ferritic-martensitic structures by cold rolling, which includes both $\gamma$-austenite and $\varepsilon$ - and $\alpha^{\prime}$-martensite.

Its extraordinary lightweight potential and corrosion resistance have led to a high market penetration of 1.4310 in the branch of spring manufacturing. Due to the benefits of the mechanical properties on the processing and the corrosion resistance on the usage, quite some not corrosionresistant or coated spring strips have been substituted by 1.4310. By now, it has reached a dominant market share of $60 \%$ in German spring manufacturing [4]. 


\section{Spring Manufacturing}

Resource-efficient spring manufacturing processes. Based both on energetic and economic reasons, springs are commonly fabricated by cold forming. Even though the term is not standardized, industry refers to the utilized manufacturing processes as the stamping-bending technology. Using progressive dies on high-speed mechanical stamping presses or special stampingbending machines, e.g. the Bihler technology, different punching and forming techniques can be combined during one working step and one stroke of the ram. [5]

For a profitable and resource-efficient production of springs, manufacturers have to focus on factors such as the utilization of material, high output per time unit and ensuring of reliable production processes. The utilization of material is mainly a question of the specific spring design and of minimizing the punching scrap. The last two factors are mainly influenced by the machine selection and the knowledge of factors influencing process deviations. Downstream, possible disruptions of the production processes have to be taken into account, e.g. due to necessary adjusting of the tools. The achievable part output is mainly predetermined by the amount of strokes per minute, which itself depends on the options of the machine and the height of stroke necessary both for the handling of the strips and the stamping of the springs. Some stamping-bending machines reach stroke rates of up to $2000 \mathrm{spm}$ when realizing only small heights of stroke. [6] Yet, the chosen process parameters are not only limited by the technical possibilities of the stampingbending machine and the tool kinematics, but to an even higher degree by insufficient knowledge of the influence of different process parameters on the reliable outcome of the bending accuracy.

Springback in bending. One effect of high influence on the dimensional accuracy during bending [7] (and all other forming operations) at room temperature is springback. It inevitably occurs during any forming processes due to the elastic proportion in the material behavior. Thus, in order to develop suitable compensation strategies and to achieve stable dimensions of manufactured parts, in-depth knowledge of the springback phenomenon and influencing factors is mandatory. Even though there are different empirically or analytically determined methods to estimate springback for materials with low tensile strengths (e.g. formulae or tables which date back to the 1950s [8-11]), a lack of accuracy often hinders a fast incorporation of tools and production processes. Reliable standard methods are not available for ultra-high strength spring strips with tensile strengths beyond $T S=1200 \mathrm{MPa}$ as nearly all related research activities are driven by automotive industries. Dimensional inaccuracies frequently lead to cost-intensive reworking of tools or tool active parts which are thus partly realized in an adjustable way. This requires both the design of complex and expensive stamping tools and iterative incorporation of the tools in the physical state, which is necessary to reach the required dimensions and process capability indices $c_{p}$.

Springback is already a big issue when processing relatively soft sheet materials; it is trending to an even enhanced importance when processing ultra-high strength spring strips. The highly elastic material behavior, which is essential during the application phase as a spring, makes a production that fulfils narrow tolerance requirements.

Generally speaking, three groups of parameters that can partly be controlled during process design are considered of having an influence on the specific occurrence of springback, namely geometric, material and process parameters.

Geometric influences include the specific geometrical layout of the bending punches, e.g. the bending core radii and the clearance between the bending core and the punch considering the tools, and bending angles, sheet widths and thickness considering the parts. Generalized rules indicate that the expected springback reduces with increased sheet thicknesses and increased bending radii.

The elastic material behavior has already been named as one of material parameters affecting springback. Dependencies especially exist to mechanical parameters such as yield strength (YS), tensile strength (TS), Young's Modulus $(E)$ and the strain hardening coefficient $(n)$. Growing yield and tensile strengths and reduced Young's modulus lead to increased springback. [12]

The third column of influencing parameters is the forming process itself. Examples that have to be mentioned include the utilized machine, e.g. precision of the ram movement or oscillations, the 
specifics of the utilized bending process, e.g. punch pressure, and the rate of forming which correlates with the punch kinematics and stroke rate. Another aspect is the orientation of the bending axis compared to the rolling direction of the sheet material, which attributes to different outcomes due to anisotropic material behavior. [13]

Published investigations and research results concerning springback in sheet metal forming of car-body components generally end at tensile strengths of $T S=1100-1200 \mathrm{MPa}$; so do empirical and analytical calculation rules mentioned in the literature. Springback has been a topic of intensive research activities over the past years. However, there is still a wide gap between the strength class of sheets used in the automotive industry and the advanced sheets in the class of ultra-high strength spring strips used in the spring industry. The lower sheet thicknesses $t=0.1-0.4 \mathrm{~mm}$ add to the uncertainties.

This sets the starting point of the experimental investigation reported in this paper. A broad variation of possible influencing parameters during bending of ultra-high strength spring bands is conducted. It is supposed to reproduce the spreading of geometric, material and process parameters in stamping-bending operations during spring manufacturing. The investigation aims at a deeper insight into the sensitivity and the effects of varied material parameters, stroke rates and other relevant process parameters during bending in spring manufacturing.

\section{Materials}

Research has been conducted with different coils of an austenitic stainless steel 1.4310 (X10CrNi18-8) which differed in their chemical compositions, their mechanical values and their micro structure / grain size. The sheet metal thickness was set at $t=0.3 \pm 0.01 \mathrm{~mm}$ and the width at $w=50.0 \pm 0.25 \mathrm{~mm}$ for each of the coils. As shown in Table 1, the chemical composition of these three materials was within the allowance specified in DIN EN 10151 [14]. While material 1 (M1) was purchased from stock, materials 2 (M2) and 3 (M3) were specifically rolled from one mother coil in order to get diverging mechanical properties in spite of an absolutely identical chemical composition. The spread of the molybdenum quantity is the most obvious difference between the materials, which attributes to the corrosion resistance and the formation of ferrite. [15] The austenitic grain size of materials M1 and M2 was 8-9, while material M3 had a finer austenitic grain size of 9-10 (referring to ASTM E 112 [16]).

Table 1. Chemical composition in weight percentage of the used materials

\begin{tabular}{|c|c|c|c|c|c|c|c|c|c|}
\hline & $\mathbf{C}$ & $\mathbf{S i}$ & $\mathbf{M n}$ & $\mathbf{P}$ & $\mathbf{S}$ & $\mathbf{C r}$ & $\mathbf{M o}$ & $\mathbf{N i}$ & $\mathbf{N}$ \\
\hline Allowance & $0.05-0.15$ & $\leq 2.00$ & $\leq 2.00$ & $\leq 0.045$ & $\leq 0.05$ & $16-19$ & $\leq 0.80$ & $6-9.5$ & $\leq 0.11$ \\
\hline M1 & 0.11 & 0.91 & 1.21 & 0.027 & 0.001 & 16.8 & 0.03 & 6.5 & 0.067 \\
\hline M2 / M3 & 0.1 & 1.18 & 1.15 & 0.027 & 0.001 & 16.9 & 0.66 & 6.4 & 0.072 \\
\hline
\end{tabular}

The target strengths of the coils were in the range $T S=1600-1800 \mathrm{MPa}$ with material M1 chosen close to the lower limit of that range, and materials M2 and M3 close to the upper limit. This variation of different materials purchased under an identical denomination was consciously chosen to attribute to the industrial practise of being able to set a certain range of the strengths of a material to be processed, but commonly not knowing details about the backgrounds of a material for sale.

Material characterizations of all three materials were undertaken to verify the general information given by the supplier. These characterizations included uniaxial tensile tests, tension-compression tests and a structural examination by optical means and by scanning electron micrograph (SEM). The observed mechanical properties are summarized in Fig. 1a.

All three examined materials show highly deformed austenite with a high percentage of approximately $60 \% \alpha^{\prime}$-martensite in its microstructure. In the longitudinal direction, the banding resulting from the cold rolling catches is clearly visible. The grain boundary seems free of precipitations. An impression from the microstructural analysis of material M1 is given in Fig. $1 \mathrm{~b}$. 


\begin{tabular}{|l|c|c|c|}
\hline a) & M1 & M2 & M3 \\
\hline YS [MPa] & 1445 & 1545 & 1556 \\
\hline TS [MPa] & 1612 & 1775 & 1824 \\
\hline UE [\%] & 4.7 & 1.3 & 1.1 \\
\hline A80 [\%] & 5.3 & 2.3 & 2.1 \\
\hline
\end{tabular}

b)

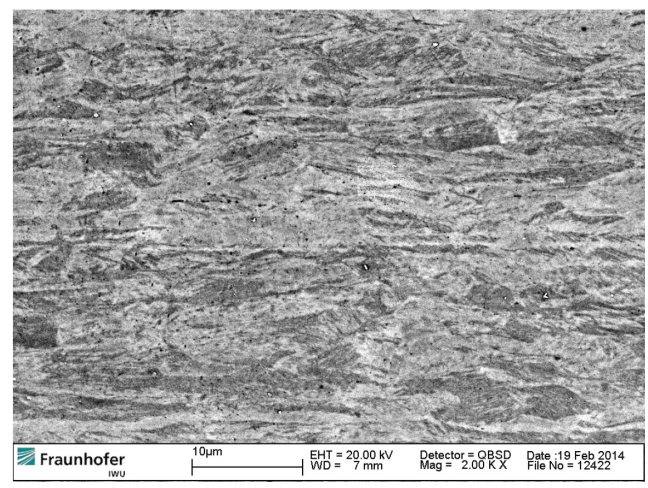

Fig. 1. a) Mechanical properties of the three test materials; b) SEM image of material M1, magnification $2000 \mathrm{x}$

\section{Experimental}

Machine. A high performance stamping press Bruderer BSTA 40 was chosen for the experimental investigations. It is a mechanical press with an eccentric drive and a nominal stamping force of $F=400 \mathrm{kN}$. Depending on the adjustable height of stroke, it is capable of realizing up to $1200 \mathrm{spm}$. The periphery of this stamping press consists of a decoiler, a straightener, a mechanical roller feed BBV 190/85 with oscillatory rollers and different sensors for monitoring the stamping forces and the penetration depth as well as a double sheet control.

Test geometry. The springs for the experimental investigations were designed to each contain three legs which are oriented parallel, perpendicularly and diagonally to the rolling and feed direction of this material. This approach allows an examination of anisotropic material behavior while measuring and evaluating one spring at a time. For labelling the three legs, as can be seen in Fig. 3a, the following specification has been met, which nominally refers to the orientation of the bending edge compared to the feed direction: The leg, which is oriented parallel to the feed direction, is labelled $90^{\circ}$ (as the bending edge is oriented perpendicularly to the feed direction), the leg oriented perpendicularly to the feed direction is labelled $0^{\circ}$ and the leg oriented diagonally to the feed direction is labelled $45^{\circ}$.

The three legs have a flat length of $l=10 \mathrm{~mm}$ and a width of $w=5 \mathrm{~mm}$. Bending conditions can principally be varied by changing bending punches. However, it was agreed to set the bending radii unvaried at $R_{b}=1.5 \mathrm{~mm}$. The resulting unvaried ratio of bending radius to sheet thickness is $R_{b} / t=5$. Besides, each of the test springs contains four stamped holes with diameters of $D=2.5 \mathrm{~mm}$ which are needed for precise positioning during the measuring.

Tool setup. A linear strip layout containing eight production steps and three empty steps was set up for the progressive die (Fig. 2): In step 1 six holes are punched which are divided into two seeker holes needed for a high feeding and repeating accuracy and the four holes within the test parts needed for the measuring position. While step 2 is left empty, steps 3 and 4 contain all stamping operations necessary to expose the legs to be bent. By providing three ridges, the test springs remain fixed with the punch scrap. Another two empty steps (5-6) were followed by three steps (7-9) available for the bending operations. In the final two steps the tests springs are separated from the punch scrap (10) before the scrap is chopped (11). The realized feed is $f=50 \mathrm{~mm}$ per stroke.

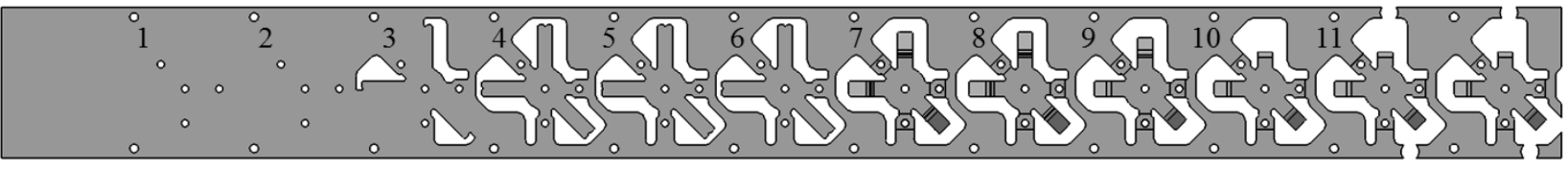

Fig. 2. Linear strip layout used in the progressive die

Different bending punches, which allow an evaluation of differing bending conditions and bending concepts, were designed and manufactured. This concept allows a rather easy change of active tool parts during the course of the experiments. The bending punches were designed in such a 
way that the named bending angles $\alpha_{n o m}$ describe the angle before springback. Values are different after recovery due to emerging springback (angle after springback). This approach differs from industrial proceedings where the angle before springback merely is a technological value needed to reliably achieve a specified tolerance zone surrounding a certain angle in the spring geometry.
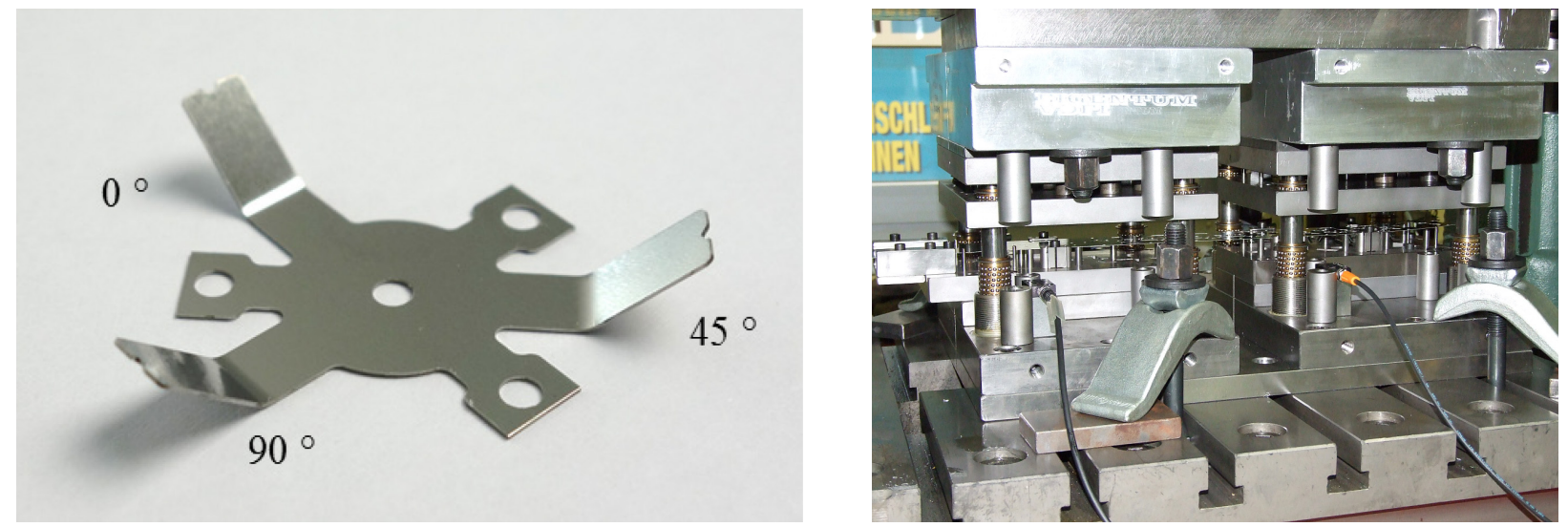

Fig. 3. a) Test part with labelling of the three legs; b) progressive die in the experimental setup

Experimental design. Table 2 summarizes the variation of parameters which have been included in the experimental investigation. Experimental runs with duration of $t=15$ minutes were conducted in order to achieve industry-oriented processing conditions. During each experimental run, enough springs were separated so that at least 30 springs of each parameter combination could be fully measured, targeting a statistical analysis of the results.

Table 2. Variation parameters for the experimental investigations

\begin{tabular}{|l|l|}
\hline & Variation parameter \\
\hline Material & M1 \\
& M2 \\
& M3 \\
\hline Orientation bending axis - & O1 $0^{\circ}$ \\
rolling direction & O2 $90^{\circ}$ \\
& O3 $45^{\circ}$ \\
\hline Bending concept & B1 $60^{\circ}$ free bending \\
& B2 $60^{\circ}$ free bending with superimposed compression on the bending radius \\
& B3 two step bending $45^{\circ} \rightarrow 60^{\circ}$ \\
\hline Stroke rate & V1 100 spm \\
& V2 $200 \mathrm{spm}$ \\
& V3 $250 \mathrm{spm}$ \\
\hline
\end{tabular}

Measuring concept. The measuring of all examined springs was conducted with a 3D-tactile measurement system Zeiss Prismo 7 S-ACC VAST on a measuring device which is capable of holding ten test springs at a time. For this purpose, the probing force of the measuring system was systematically reduced until no deflection of the bent legs could be observed during measurement. The probing force was ultimately set at $F_{p}=100 \mathrm{mN}$. Repeat accuracy of the measured angles was determined as $0.04^{\circ}$. This value was raised in 30 consecutive measurements, each involving assembly and disassembly of the probed spring on the measuring device. Measured and recorded values include the diameters of the punched holes, the flatness of the base, the angles of the bent legs after springback and the length of the legs.

Statistical analysis. Results of the measuring are angles $\alpha_{S b}$ after springback emerged. Compared to the nominal angle $\alpha_{\text {nom }}$ of the tool active parts, the measured springback angles $\Delta \alpha$ can be calculated with formula (1):

$$
\Delta \alpha=\alpha_{n o m}-\alpha_{S b}
$$


For comparison of the springback values, the arithmetic mean $\overline{\Delta \alpha}$ of the springback angles $\Delta \alpha$ of each data set is to be calculated with (1):

$$
\overline{\Delta \alpha}=\frac{1}{n} \cdot \sum_{i=1}^{n} \Delta \alpha_{i}
$$

Another statistical approach for looking at the springback angles consists of evaluating their statistical dispersion within a data set. While considering (1) and (2), the standard deviation $s_{\Delta \alpha}$ as the chosen statistical value is calculated as follows:

$$
s_{\Delta \alpha}=\sqrt{\frac{1}{n} \cdot \sum_{i=1}^{n}\left(\Delta \alpha_{i}-\overline{\Delta \alpha}\right)^{2}}
$$

\section{Results}

Influence of the materials. The three tested materials do have a major influence on the observed springback. Within the parameter variation, no universally valid correlation between the materials and the springback could be found, especially when considering the obvious anisotropic material behavior.

Fig. 4 presents two diagrams comparing materials M1, M2 and M3, using free bending (B1), which show significant differences in springback angles $\overline{\Delta \alpha}$. For the orientation $\mathrm{O} 1$ (bending edge oriented parallel to the feed direction) material M2 shows by far the highest amount of springback. In addition, there is a strong dependency of the stroke rate with values at V1 (100 spm), exceeding the values of $\overline{\Delta \alpha}$ at V3 $(250 \mathrm{spm})$ by more than $1.2^{\circ}$. The same general tendency can be seen for material M3 which has a $1.2^{\circ}$ lower springback $\overline{\Delta \alpha}$ than material M2 at the stroke rate V3 and an even higher difference of nearly $1.9^{\circ}$ at stroke rate V1. It is a general observation that can be seen over most of the data sets that the springback $\Delta \alpha$ reduces with increasing stroke rates. Main influences leading to this phenomenon might be changing dynamics of the process and possibly even strain rate dependencies of the spring strips. Material M1 is a bit of an outlier in this interpretation as there is indeed a slight increase in springback $\Delta \alpha$ of $0.3^{\circ}$. This data set corresponds quite well with what can be expected due to the mechanical properties. Material M1 with the lowest yield strength and highest Young's modulus shows the lowest springback while the measured springback angles of materials M2 and M3 are up to $3^{\circ}$ higher than those of material M1. Apparently, material M3 seems to tend a bit less towards springback for orientation O1, which might attribute to the finer grain size.
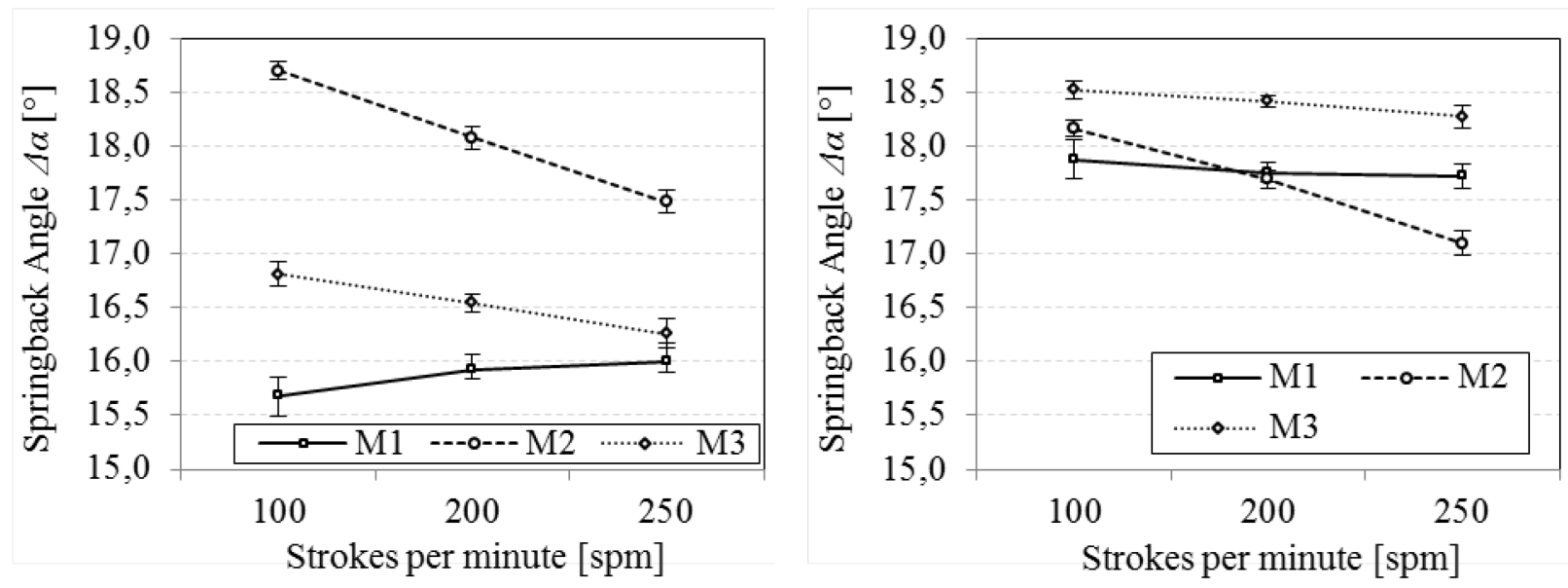

Fig. 4. Observed absolute values of springback and calculated standard deviations for the free bending (B1): orientation O1 (left), orientation O2 (right) 
The results are different when looking at the same bending concept, but with a different orientation, namely $\mathrm{O} 2$, which is oriented perpendicularly to the rolling direction. The impressive finding is that springback $\Delta \alpha$ for the materials M1 and M3 is $1.7-2.0^{\circ}$ above the springback reported for orientation $\mathrm{O} 1$. In contrast, the springback for material M2 is slightly reduced by $0.3-0.5^{\circ}$, yet showing a similar trend when additionally looking at the stroke rates.

While the absolute amount of springback varies depending on the processed materials, the differences in the standard deviations $s_{\Delta \alpha}$ are less distinct with a slightly higher statistical dispersion for material M1 compared to materials M2 and M3. For the two specified examples, the standard deviations $s_{\Delta \alpha}$ varied between $0.08^{\circ}$ and $0.18^{\circ}$.

Influence of the orientation of the bending axis compared to the rolling direction. A strong anisotropic material behavior results from the cold rolling of the materials. Fig. 5 presents the example of two data sets. The first diagram shows a two-step bending, bending concept B3, in combination with material M2. The springback $\Delta \alpha$ of the orientations $\mathrm{O} 1$ and $\mathrm{O} 2$ with bending axis respectively oriented parallel and perpendicularly to the rolling direction shows little difference. Once again there is a slight tendency that springback reduces with increased stroke rate. The orientation $\mathrm{O} 3$ with the bending axis oriented diagonally to the rolling direction shows a significant lower springback $\Delta \alpha$ with values approximately $2^{\circ}$ lower than in the other two directions. This cannot be explained in detail, but might be attributed to the fact that in bending in the directions $\mathrm{O} 1$ and $\mathrm{O} 2$, the banding of the grains resulting from the cold rolling of the materials has to be taken into account additionally.
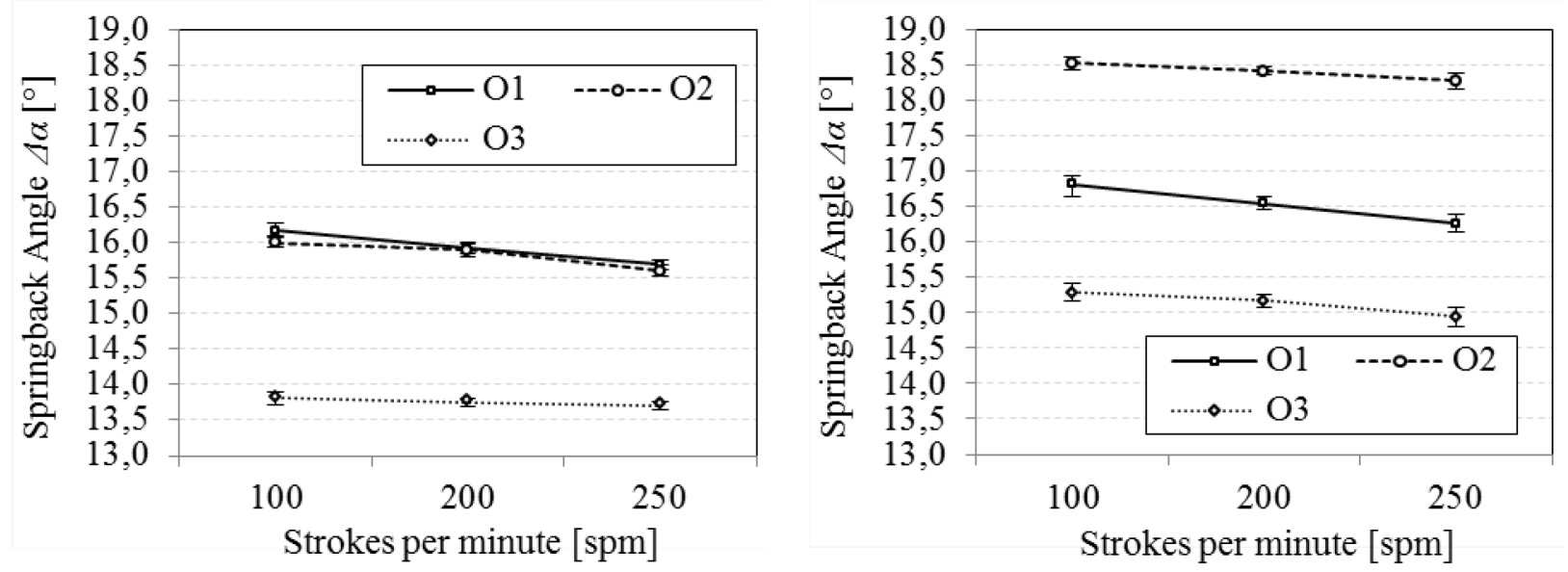

Fig. 5. Observed absolute values of springback and calculated standard deviations for the two-step bending (B3) of material M2 (left) and of the free bending B1 with material M3 (right)

In the second example, which combines the bending concept free bending B1 and the material $\mathrm{M} 3$, the influence of the orientation of the bending axis compared to the rolling directions becomes even more distinct. In absolute values there is a difference in springback angles $\Delta \alpha$ of $1.7-2.0^{\circ}$ between the orientations $\mathrm{O} 1$ and $\mathrm{O} 2$. The orientation $\mathrm{O} 3$ with the bending axis oriented diagonally to the rolling direction once again shows the smallest amount of springback which was measured as $\Delta \alpha=14.9-15.3^{\circ}$. All parameter sets show the tendency that springback reduces with higher stroke rates.

Influence of bending concept. The three bending concepts show significant impact on the amount of springback Fig. 6 illustrates the springback of two orientations compared to the rolling direction of material M2, and the bending concept B1 (free bending) shows the highest amount of springback at all considered stroke rates. The bending concepts B2 (free bending with superimposed compression) and B3 (two-step bending) proved to be advantageous regarding the absolute amount of springback. This virtually corresponds to the industrial practice of mainly implementing the last two mentioned bending concepts in production processes. Presumably, the observed results can be attributed to the specifics of the bending processes that exploit advanced strain-hardening. In the 
bending concept B2 this is achieved by the additional compression applied on the bending radius. In the bending concept B3 the bending mechanism contains a strain-hardening in the first forming step which is followed by relief. The differences in the springback angles $\Delta \alpha$ between the three bending concepts are as high as $2.2^{\circ}$.
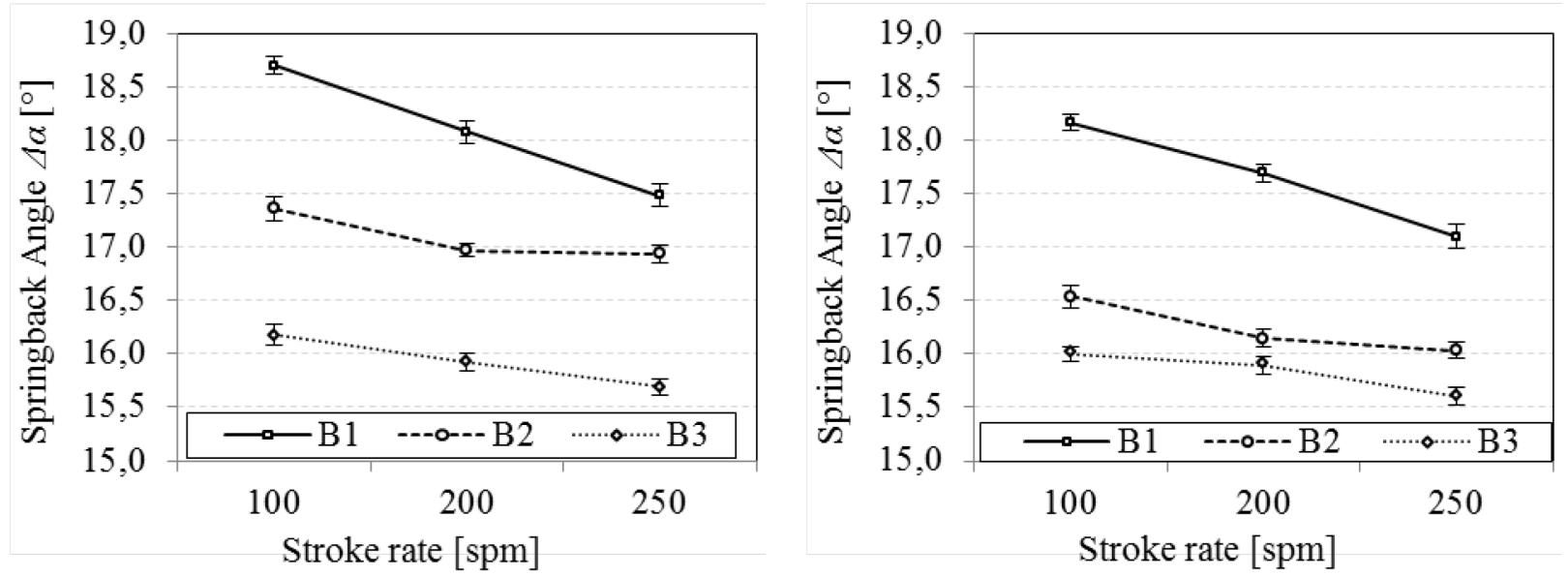

Fig. 6. Observed absolute values of springback and calculated standard deviations for the bending of material M2 and orientation $\mathrm{O} 1$ (left) and orientation $\mathrm{O} 2$ (right)

\section{Summary and Outlook}

In this experimental study the springback occurring during bending operations of ultra-high strength spring strips has been examined. A total of 27 parameter combinations of different materials, bending concepts and stroke rates have been considered during experimentation. Different parameters, e.g. materials, orientation of the bending axis compared to the rolling direction and the chosen bending concept proved to be significant impact factors affecting the springback. Not all the results proved to be absolutely explainable, especially seeing random distributions of the springback dispersion. This all adds to the uncertainties in industrial production processes, where small changes in the process condition might easily change springback beyond tolerable values, thus making adjustments necessary.

In order to obtain deeper knowledge and understanding of the influencing parameters in springback behavior of ultra-high strength spring strips, the simulation-based prediction of spring manufacturing processes will be the subject of future research activities. Based on the presented experimental results, the exemplary bending process will be modelled and computed using an appropriate FE simulation system. In combination with intensified and advanced material testing and modelling, the numerical prediction of springback behavior will be investigated in detail to achieve suitable strategies for industrial tool design while processing ultra-high strength spring strips.

\section{Acknowledgement}

The work presented in this paper has kindly been funded by the Arbeitsgemeinschaft industrieller Forschungsvereinigungen „Otto von Guericke“ (grant numbers: IGF 17423 BG and IGF 19273 BR). The projects have been accompanied by the German Association of Spring Manufacturers (VDFI). 


\section{References}

[1] R. Neugebauer et al., F. Schieck, S. Polster, A. Mosel, A. Rautenstrauch, J. Schönherr, N. Pierschel, Press hardening. An innovative and challenging technology, Arch. Civ. Mech. Eng. 12 (2012) 113-118.

[2] M. Meissner, H.-J. Schorcht, Metallfedern. Grundlagen, Werkstoffe, Berechnung, Gestaltung und Rechnereinsatz, second ed., Berlin / Heidelberg / New York, 2007.

[3] W. Hellwig, M. Kolbe, Spanlose Fertigung Stanzen. Integrierte Fertigung komplexer Präzisions-Stanzteile, tenth ed., Wiesbaden, 2012.

[4] Information on http://www.federnverband.de

[5] V. Hörmann, C. Schäfer, Stanzbiegetechnik. Effiziente Fertigung von Stanzbiegeteilen und kompletten Baugruppen. München 2013.

[6] Information on: http://www.bruderer.com

[7] T.X. Yu, L.C. Zhang, Plastic bending: Theory and Application, World Scientific Publishing, Singapore, 1996.

[8] R. Boklen, Nomogramm zur Bestimmung des Spannungsverlaufes bei reiner Biegung für Werkstoffe mit linearer Verfestigung, Zeitschrift für Metallkunde, 44 (1953) 8, 382-386.

[9] F.J. Gardiner, The springback of metals. Transactions of the AIME 79 (1957), 1-9.

[10] W.P. Romanowski, Handbuch der Stanztechnik, Verlag Technik Berlin, 1959.

[11] R.H.Wagoner, H. Lim, M.-G. Lee, Advanced Issues in springback, Int.J. of Plast.45(2013)3-20.

[12] W.F. Hosford, R.M. Caddell, Metal Forming. Mechanics and Metallurgy, third ed., Cambridge University Press, Cambridge 2007.

[13] M. Krinninger, D. Opritescu, R. Golle, W. Volk, Experimental investigation of the influence of punch velocity on the springback behavior and the flat length in free bending. Procedia CIRP 41 (2016) 1066-1071.

[14]DIN EN 10151, Stainless steel strip for springs - Technical delivery conditions, 2003

[15] Information on http://www.edelstahlrostfrei.de/downloads/ISER/Wirkungsweise_wichtiger_Legierungselemente.pdf

[16] ASTM E112-13 Standard Test Methods for Determining Average Grain Size, 2013. 